\title{
Reversible retiform purpura: a sign of cocaine use
}

\author{
Christina Han MD, Gayatri Sreenivasan MD, Jan P. Dutz MD
}

$\mathrm{A}$ 52-year-old woman with a history of frequent inhalational cocaine use was admitted to hospital after presenting to the emergency department with a four-month history of intermittent painful bruises (retiform purpura) involving her arms and legs, with recent ulceration of her nasal tip, ears and cheeks (Figures 1A, 2A and $3 \mathrm{~A})$. Previous episodes of the retiform purpura had improved spontaneously following abstinence from inhalational cocaine use. The patient's medical history included Parkinson disease and emphysema. She had no history of venous or arterial thromboembolism, pregnancy complications or spontaneous abortions.

A complete blood count showed a leukocyte count of 2.4 (normal 4.0-11.0) $\times 10^{9} / \mathrm{L}$, with a low absolute neutrophil count of 1.4 (normal $2.0-8.0) \times 10^{9} / \mathrm{L}$, a hemoglobin level of 112 (normal 115-155) g/L and a normal platelet count of 184 (normal 150-400) $\times 10^{9} / \mathrm{L}$. A positive atypical perinuclear antineutrophil cytoplasmic antibody (titer $>1: 640$ ) was detected, with an elevated proteinase 3 level. Testing for anticardiolipin antibodies was positive for immunoglobulin M (level 37.3 [normal 0-20] MPU) and negative for immunoglobulin G. $\beta_{2}$ glycoprotein I antibody and lupus anticoagulant testing was negative. International normalized ratio, partial thromboplastin time, and protein $\mathrm{C}$, protein $\mathrm{S}$ and antithrombin levels were normal, as were the results of complement, cryoglobulin, rheumatoid factor, antinuclear antibody and extractable nuclear antigen tests. A skin biopsy showed extensive thrombotic vasculopathy without evidence of vasculitis. Results of echocardiography, blood culture and special stains on skin biopsy ruled out an underlying infectious vaso-occlusive process.

The patient had been admitted to another hospital three months previously with similar but less dramatic clinical findings, also with positive anticardiolipin immunoglobulin M serology of 43.2 MPU. The lesions had improved spontaneously during the patient's hospital stay. This previous admission had also been preceded by recent exposure to cocaine. Thus, with clinical features of cutaneous vascular necrosis and recurrence of abnormal anticardiolipin serology 12 weeks apart, a diagnosis of antiphospholipid antibody syndrome was made.

The patient was given pulse intravenous steroids and subcutaneous dalteparin for anticoagulation during her course in hospital. Further, abstinence from inhalational cocaine was recommended. Despite extensive purpura at presentation suggestive of necrosis, the lesions improved, demonstrating evidence of reperfusion and reversibility (Figures 1B, 3B and 3C). In addition, neutropenia reversed spontaneously, as had been noted on her previous hospital admission. The patient was given warfarin as an outpatient and maintained a therapeutic international normalized ratio of 2.0-3.0.

Despite measures to encourage abstinence from inhalational cocaine use and despite ongoing anticoagulation treatment at therapeutic levels, a third admission to hospital was required, with clinical evidence of retiform purpura of the legs (Figure 2B) and neutropenia after cocaine inhalation. Reversibility was again shown with abstinence from cocaine use. Warfarin was stopped and the patient was given dalteparin for ongoing anticoagulation therapy, but she continued to develop new skin lesions following repeated cocaine inhalation. Reinitiation of oral prednisone therapy at $20 \mathrm{mg} / \mathrm{d}$ resulted in further improvement. The unique clinical reversibility of this phenomenon on abstinence from drug use and reliable clinical recurrence with exposure to cocaine on multiple occasions is noteworthy.
Competing interests: None declared.

This article has been peer reviewed.

Correspondence to: Dr. Jan P. Dutz, dutz@interchange.ubc.ca

CMAJ 2011. DOI:10.1503 /cmaj.101005

\section{KEY POINTS}

- Patients exposed to levamisole-containing cocaine may present with skin changes including retiform purpura, livedo reticularis and, ultimately, cutaneous necrosis.

- Reversibility and reperfusion may be achieved with discontinuation of cocaine use, but anticoagulation, immunotherapy or both may be required.

- If patients have recently used cocaine, exposure to levamisole should be considered in those with sudden-onset retiform purpura, neutropenia, and positive testing for antineutrophil cytoplasmic autoantibodies and antiphospholipid antibodies. 


\section{Discussion}

Retiform purpura present as irregular nonblanching violaceous plaques, reminiscent of a net-like pattern. A skin biopsy frequently shows substantial intravascular occlusion without vasculitis, termed vasculopathy. The differential diagnosis can be extensive. Box 1 provides a list of differential diagnoses to consider in the presentation of retiform purpura. ${ }^{1}$ Antiphospholipid antibody syndrome is characterized by an occlusive vasculopathy secondary to a lupus anticoagulant, anticardiolipin or $\beta_{2}$ glycoprotein I antibodies and is a common cause of retiform purpura. This disorder may be triggered by underlying infection, drugs, surgery and genetic predisposition.

\section{Cocaine and levamisole}

Cocaine contaminated with levamisole may be a new cause of retiform purpura. Five patients with retiform purpura involving the ears, cheeks or both after cocaine exposure were described in the recent literature. ${ }^{2-4}$ These reports showed striking clinical similarity, with tender purpuric plaques and areas of central necrosis affecting the ears, cheeks and often the extremities. An identical
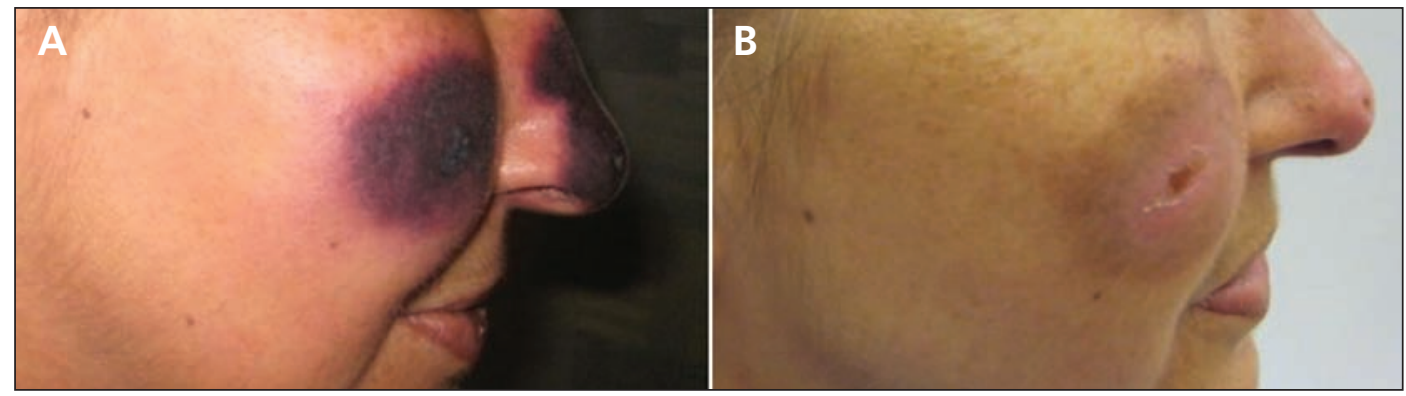

Figure 1: Cutaneous necrosis of the cheek and nose in a 52-year-old woman (A) at initial presentation and (B) 20 days later.
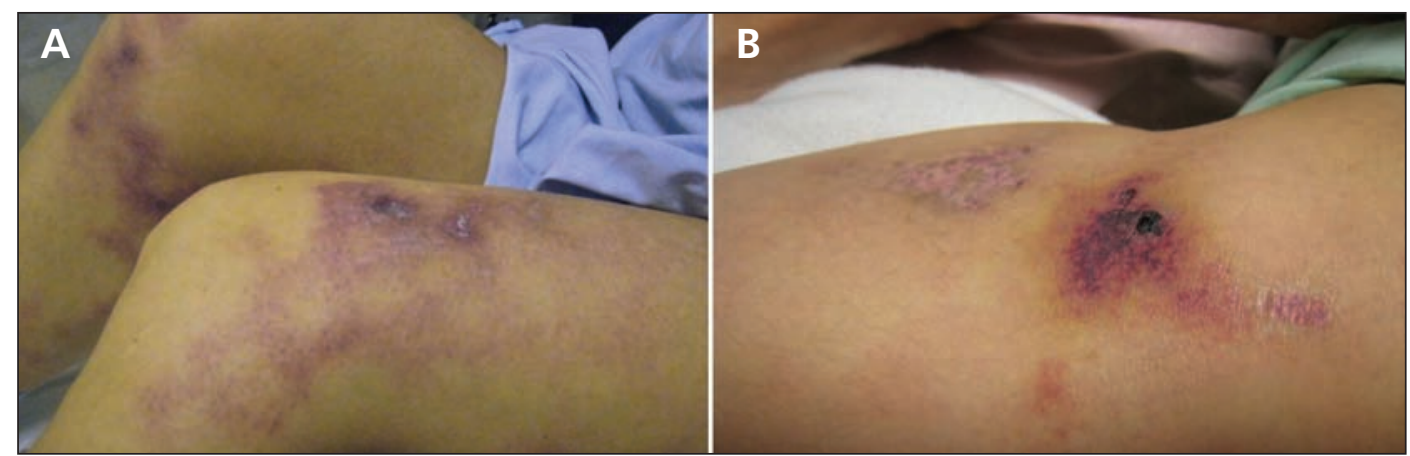

Figure 2: Livedoid pattern with ulceration on the legs (A) at initial presentation and (B) new lesions on readmission 53 days later.

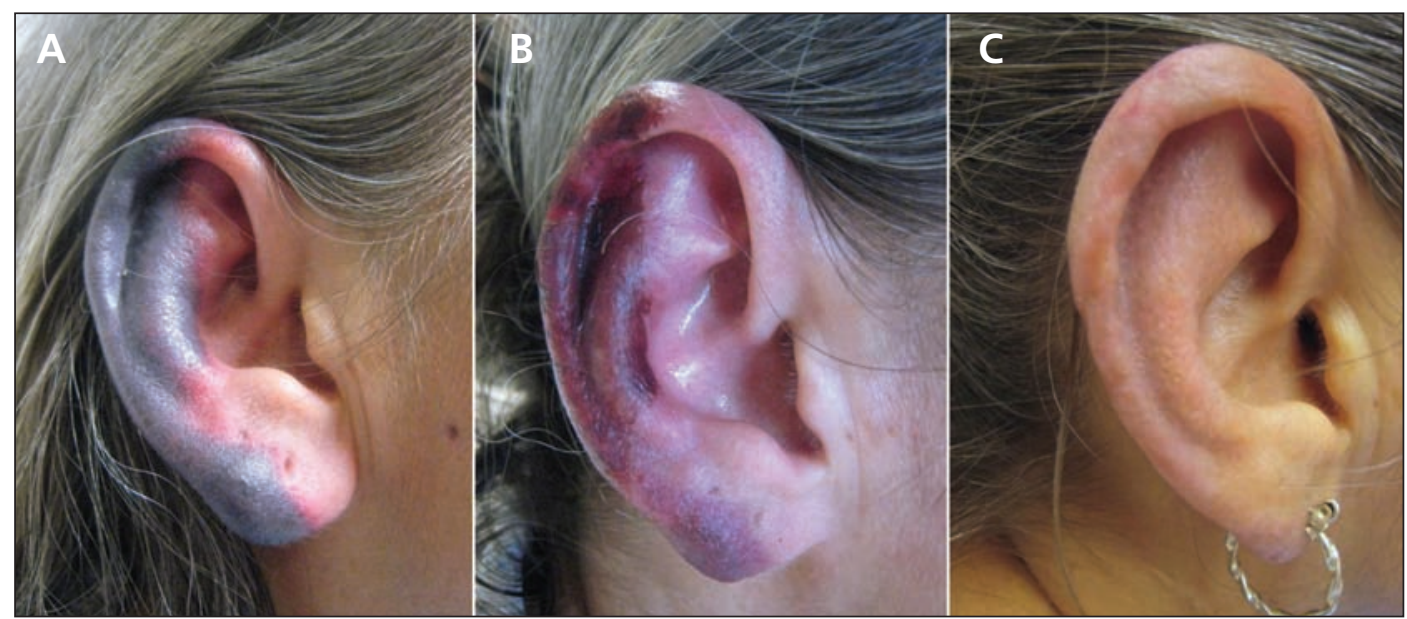

Figure 3: Right ear of the patient (A) at inital presentation, (B) 7 days later following treatment and (C) 19 days after the initial presentation, showing reversible cutaneous necrosis. 
presentation was described in children exposed to levamisole only. ${ }^{5,6}$ Two patients presenting with retiform purpura affecting mainly the legs after cocaine use have also been recently described. Urine toxicology in these two patients showed levamisole shortly after binge cocaine use. Persistent neutropenia was also reported, with concomitant positive testing for antineutrophil cytoplasmic autoantibodies and either positive lupus anticoagulant or anticardiolipin immunoglobulin M levels. Skin biopsy results were consistent in showing intravascular thrombi. The presence of a leukocytoclastic vasculitis was variable.

Historically, levamisole has been used in humans for its immunomodulatory properties in the treatment of various autoimmune diseases, nephrotic syndrome and neoplasms. Currently, levamisole remains widely used as an anthelmintic veterinary agent and has been increasingly found as a contaminant and possible additive to the North American cocaine supply since 2007. ${ }^{8}$ As of $2009,46 \%$ of cocaine tested at the drug analysis laboratory of Health Canada in British Columbia tested positive for levamisole, ${ }^{9}$ and $71 \%$ of the supply in the United States was adulterated with levamisole according to a recent publication by the US Department of Justice. ${ }^{8}$ It is speculated that levamisole may be added to enhance and prolong the effects of cocaine, by potentially inhibiting presynaptic catecholamine reuptake. ${ }^{10}$

\section{Neutropenia}

Neutropenia following levamisole use has been well-documented since the 1970s, and increasing reports of severe agranulocytosis have been described following exposure to levamisolecontaminated cocaine. ${ }^{9,11,12}$ This potential adverse effect resulted in a nationwide public warning by the Substance Abuse and Mental Health Services Administration in September 2009 and increased our suspicion of adulterated cocaine use in this patient.

\section{Mechanism}

The mechanism by which levamisole induces retiform purpura with intravascular thrombosis in certain individuals, and in such a unique distribution, most consistently affecting the ears, is poorly understood. Although several instances of levamisole-induced vasculitis were documented in the literature, ${ }^{5,6,13-17}$ true vasculitis was not reliably shown in all instances, and patient risk factors such as pre-existing thrombophilic states were not discussed.

Czuchlewski and coauthors identified 20 instances of unexplained agranulocytosis in New Mexico and British Columbia, and attributed
14 instances (70\%) to recent use of levamisoletainted cocaine. ${ }^{11}$ Increased bone marrow plasma cells and circulating plasmacytoid lymphocytes were found in the cocaine-exposed group, which is suggestive of an antibody-mediated process. Furthermore, $60 \%$ of patients with cocaine-associated agranulocytosis tested positive for human leukocyte antigen B27, which is consistent with previous reports that suggested an increased susceptibility to levamisole-induced agranulocytosis in this population. ${ }^{18}$ Whether antibodies and genetic susceptibility can be translated to the presentation of retiform purpura and cutaneous necrosis remains unclear. We postulate that the vasoconstrictive effect of cocaine combined with the possible autoantibody-promoting ability of levamisole may explain both the clinical distribution and unique reversibility of this phenomenon. A comparison between autoantibody profiles in users of cocaine that is either adulterated with levamisole or not may shed further light on this hypothesis.

The half-life of levamisole is relatively short (5.6 hours),$^{19}$ and its identification requires special measures including gas chromatography and mass spectrometry, which makes its detection challenging. Given our patient's clinical findings of reti-

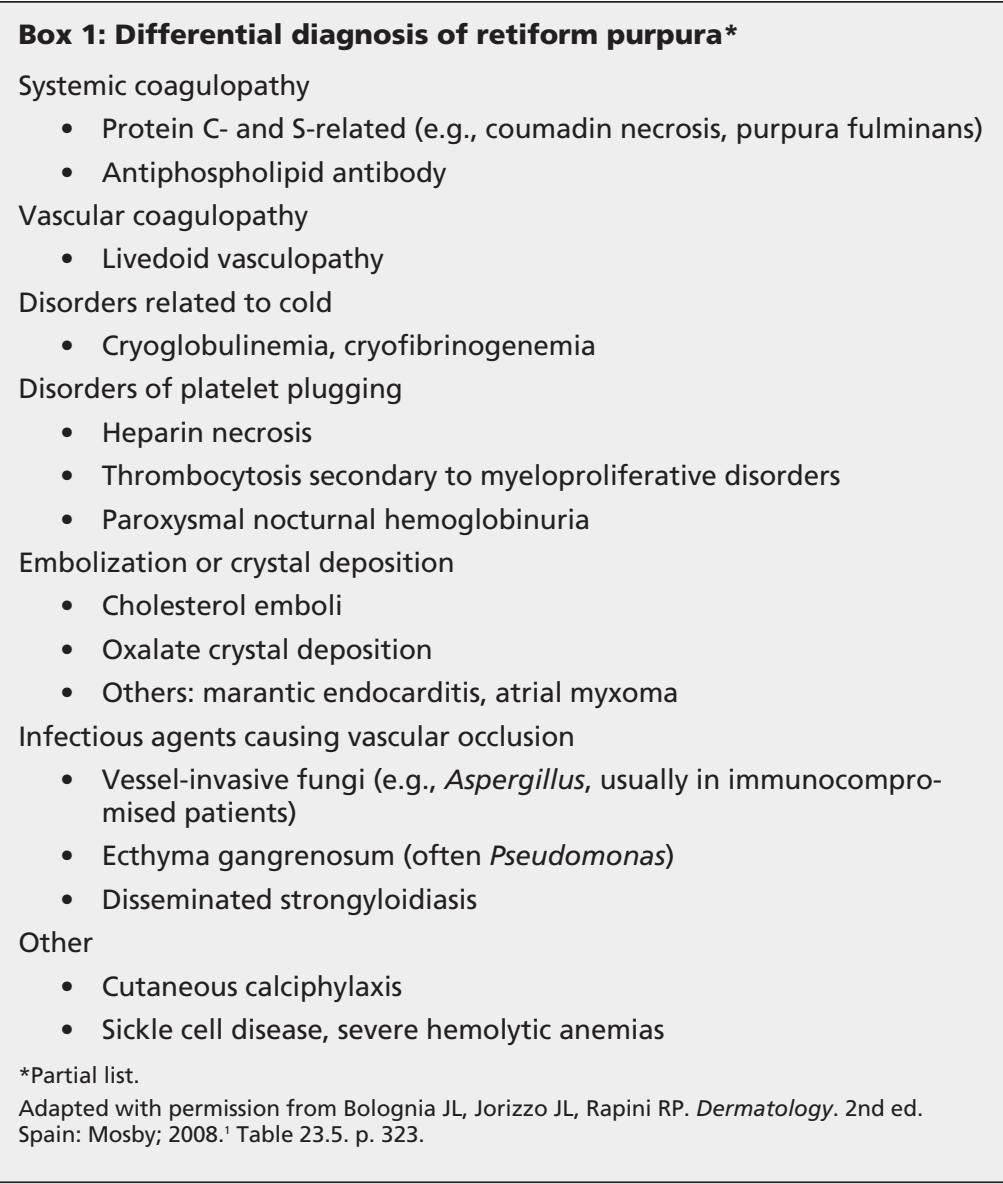


form purpura, supportive laboratory findings (positive testing for anticardiolipin and antineutrophil cytoplasmic autoantibodies, and neutropenia) as well as a temporal association with cocaine use, we strongly suspect exposure to levamisole as a potential trigger to an underlying genetic propensity for antiphospholipid antibody production. We propose that the intermittent nature of the retiform purpura as demonstrated in this patient, and repeated cutaneous necrosis and neutropenia associated with recurrent cocaine exposure are unique features of antiphospholipid antibody syndrome induced by cocaine and levamisole.

The relatively ubiquitous contamination of North American cocaine with levamisole as well as the clinical findings of retiform purpura and neutropenia should prompt physicians to consider use of adulterated cocaine as a cause of this relatively new phenomenon. Based on our review of the literature and experience with this patient, we suggest that toxicological testing for cocaine and levamisole should be considered in patients with retiform purpura. Abstinence from cocaine, anticoagulation and corticosteroid use may improve the clinical outcome.

\section{References}

1. Bolognia JL, Jorizzo JL, Rapini RP. Dermatology. 2nd ed Spain: Mosby; 2008. p. 323.

2. Bradford M, Rosenberg B, Moreno J, et al. Bilateral necrosis of earlobes and cheeks: another complication of cocaine contaminated with levamisole. Ann Intern Med 2010;152:758-9.

3. Waller JM, Feramisco JD, Alberta-Wszolek L, et al. Cocaineassociated retiform purpura and neutropenia: Is levamisole the culprit? [letter] J Am Acad Dermatol 2010;63:530-5.

4. Buchanan JA, Vogel JA, Eberhardt AM. Levamisole-induced occlusive necrotizing vasculitis of the ears after use of cocaine contaminated with levamisole. J Med Toxicol 2010 June 12 [Epub ahead of print].

5. Rongioletti F, Ghio L, Ginevri F, et al. Purpura of the ears: a distinctive vasculopathy with circulating autoantibodies complicating long-term treatment with levamisole in children. Br J Dermatol 1999;140:948-51.

6. Menni S, Pistritto G, Gianotti R, et al. Ear lobe bilateral necrosis by levamisole-induced occlusive vasculitis in a pediatric patient. Pediatr Dermatol 1997;14:477-9.

7. Walsh NM, Green PJ, Burlingame RW, et al. Cocaine-related retiform purpura: evidence to incriminate the adulterant, levamisole. J Cutan Pathol 2010;37:1212-9.

8. National drug threat drug assessment 2010. Washington (DC): US Department of Justice, National Drug Intelligence Center; 2010. Available: www.justice.gov/ndic/pubs38/38661/drugImpact htm (accessed 2010 June 19).

9. Wiens MO, Son WK, Ross C, et al. Cocaine adulterant linked to neutropenia. CMAJ 2010;182:57-9.

10. Gulati OD, Hemavathi KG, Joshi DP. Interactions of levamisole with some autonomic drugs on guinea-pig vas deferens. J Auton Pharmacol 1985;5:19-23.

11. Czuchlewski DR, Brackney M, Ewers C, et al. Clinicopathologic features of agranulocytosis in the setting of levamisoletainted cocaine. Am J Clin Pathol 2010;133:466-72.

12. Zhu NY, LeGatt DF, Turner AR. Agranulocytosis after consumption of cocaine adulterated with levamisole. Ann Intern Med 2009;150:287-9.

13. Bagga A, Hari P. Levamisole-induced vasculitis. Pediatr Nephrol 2000;14:1057-8.

14. Ferlazzo B, Barresi G, Puglisi A. Cutaneous necrotizing vasculitis caused by immunocomplexes during treatment with levamisole [article in Italian]. Boll Ist Sieroter Milan 1983;62:107-11.

15. Laux-End R, Inaebnit D, Gerber HA, et al. Vasculitis associated with levamisole and circulating autoantibodies. Arch Dis Child 1996;75:355-6.

16. Macfarlane DG, Bacon PA. Levamisole-induced vasculitis due to circulating immune complexes. BMJ 1978;1:407-8.

17. Scheinberg MA, Bezerra JB, Almeida FA, et al. Cutaneous necrotising vasculitis induced by levamisole. BMJ 1978;1:408.

18. Drew SI, Carter BM. Granulocytotoxic autoantibodies in HLA-B27 neutropenic patients. Transplantation 1979;27:436-7.

19. Kouassi E, Caille G, Lery L, et al. Novel assay and pharmacokinetics of levamisole and p-hydroxylevamisole in human plasma and urine. Biopharm Drug Dispos 1986;7:71-89.

Affiliations: From the Department of Dermatology and Skin Science (Han, Dutz) and the Department of Medicine (Sreenivasan), University of British Columbia, Vancouver, BC

Contributors: Christina Han and Jan P. Dutz drafted the manuscript. All the authors revised the manuscript and approved the final version submitted for publication.

Acknowledgements: Jan P. Dutz is a senior scholar of the Michael Smith Foundation and a senior scholar at the Child \& Family Research Institute, Vancouver, BC. 